\title{
Testing two techniques for wavefront analysis. Specific applications and comparative study
}

\section{Pruebas con dos técnicas para el análisis de frentes de onda. Aplicaciones concretas y estudio comparativo}

\author{
S. Vallmitjana ${ }^{1 S^{*}}$, A. Marzoa ${ }^{1 S}$, S. Bosch ${ }^{1 S}$ and E. Acosta ${ }^{2 S}$ \\ 1. Departament de Física Aplicada, Facultat de Física, Universidad de Barcelona, \\ 08028 Barcelona, (Spain) \\ 2. Departamento de Física Aplicada, Universidade de Santiago de Compostela, Facultade \\ de Óptica e Optometría (Campus Vida), 15782 Santiago de Compostela (Spain)
(*) E-mail: santi.vallmitjana@ub.edu
Received: 01/12/2016
S: SEDOPTICA member
Accepted: 18/09/2017 \\ DOI: $10.7149 /$ OPA.50.4.49022
}

\begin{abstract}
:
This work focuses on the description of two different techniques for characterizing wavefronts: by using Shack-Hartmann (SH) sensor and by using Point Diffraction Interferometer (PDI). Moreover describes application examples of each in recent research, and finally a comparative study of both techniques on a single optical system is performed.
\end{abstract}

Key words: Wavefront aberration; Optical testing; Zernike polynomials; Shack-Hartmann; Point diffraction interferometer; Image quality assesment.

\section{RESUMEN:}

Este trabajo se centra en la descripción de dos técnicas diferentes para caracterizar un frente de onda: mediante sensor Shack-Hartmann (SH) e interferometría de difracción por orificio o point-diffraction interferometer (PDI). Además detalla un ejemplo de aplicación de cada una de ellas en investigaciones recientes, y finalmente se realiza un estudio comparativo de ambas técnicas sobre un mismo sistema óptico.

Palabras clave: Aberración de frente de onda; Examen de sistemas ópticos; Polinomios de Zernike; Sensor Shack-Hartmann; Interferómetro de difracción por orificio; Evaluación de la calidad de imagen.

\section{REFERENCES AND LINKS / REFERENCIAS Y ENLACES}

[1] B. C. Platt, R. Shack, "History and Principles of Shack-Hartmann Wavefront Sensing", Journal of Refractive Surgery, 17, 5 S573-S577 (2001).

[2] P.M. Prieto, F. Vargas-Martín, S. Goelz, P. Artal, "Analysis of the performance of the Hartmann-Shack sensor in the human eye " J. Opt Soc Am (A), 17, 8 1388-1398 (2000).

[3] R. N. Smartt and W. H. Steel, "Theory and application of point diffraction interferometers," Jpn. J. Appl. Phys. 14, 351-356 (1975).

[4] E. Acosta, S. Chamadoira, R. Blendowske, "Modified point diffraction interferometer for inspection and evaluation of ophthalmic components".J Opt Soc Am (A), 23, 632-7 (2006).

[5] M. Born, E. Wolf, Principles of optics: Electromagnetic theory of propagation, interference and diffraction of light. Cambridge, Cambridge University Press (1999). 
[6] V. Lakshminarayanan et al, "Zernike polynomials: a guide," Journal of Modern Optics, 58, 545-561, (2011).

[7] V. Mahajan, "Aberration Theory made simple," SPIE PRESS Tutorial Text, Washingtonew, (1991).

[8] S. Chamadoira, "Point diffraction interferometer for inspection and characterization of progressive addition lenses," Ph. D. Dissertation, University of Santiago de Compostela, Santiago de Compostela, (2011).

[9] S. Vallmitjana, C. Ferran, S. Bosch, "Non-contact technique for testing antique optical instruments based on wavefront sensing," Journal of Modern Optics, 58:14, 11269-1277, (2010).

[10] J. Goodman, "Introduction to Fourier Optics," New York: McGraw-Hill, New York, (1996).

[11] S. Bosch et al, "Ray tracing, wavefronts and caustics: use of a Shack-Hartmann wavefront sensor for analyzing light propagation," Proceedings of SPIE, vol. 7429, 2009.

[12] Linikk, V., "Simple interferometer for the investigation of optical systems,"C.R. Acad. Sci. USSR 1, 208210 (1933).

[13] Chamadoira, S., Blendowske, R. and Acosta, E. "Progressive Addition Lens Measurement by Point Diffraction Interferometry," Optometry \& Vision Science. 89(10):1532-1542, (2012).

[14] S. Vallmitjana et al, "Point-diffraction interferometry to measure local curvatures and caustics of noisy wavefronts: Application for determining optical properties of fish lenses," Journal of European Optical Society Rapid Publications, 10, 15010, (2015).

[15] Neumeyer, C. "Color Vision in Fishes and Its Neural Basis", p 223-235, en Sensory Processing in Aquatic Environments,Springer (2003).

[16] J. M. Bueno et al, "Wavefront measurements of phase plates combining a point-diffraction interferometer and a Hartmann-Shack sensor," Applied Optics 49 (2010).

[17] Atmosfringe: http://www.atmos-software.it/AtmosFringe.html

\section{Introduction}

In different fields of science and technology the measurement and analysis of the image quality of optical systems is of capital importance and it is in vigorous and continuous development. Even though a large number of techniques are available for optical testing, interferometry and wavefront sensing techniques have been used extensively and commonly in the last decades. This work focuses in these two particular areas for the assessment of the image quality, based on the analysis of the wavefront aberration (WA): the Shack-Hartmann (SH) wavefront sensor and the Point Diffraction Interferometer (PDI).

Although the SH wavefront sensor was initially proposed for use in astronomy [1] by correcting the refractive changes introduced by atmosphere turbulence, with the help of deformable optics, has been extensively used in many areas, such as adaptive optics and ophthalmology [2]. On the other hand, in the last few years, the PDI [3] has a growing use in areas like ophthalmology because of its simplicity and high dynamical range [4].

This paper describes both techniques and shows practical applications carrying out each one in our laboratory. As example of the SH technique we have elaborated a noncontact method for determining the optical quality of antique instruments, such as a spyglass of the early twentieth century or a microscope objective from the nineteen century.

As example of PDI we have developed an experimental method for measuring local curvatures of the wavefront and then by computing, the precise location of caustics is obtained. This study was done specifically in crystalline of different species of fishes.

Finally, both methods applied on the same optical system are compared, allowing to analyze the accuracy of both, see the equivalences and the similarity of the results and highlights the advantages and disadvantages of each method. At the same time the work has been a good test to deepen our knowledge of both techniques. 


\section{Shack-Hartmann sensor and Point Diffraction Interferometer basis}

\subsection{Shack-Hartmann sensor}

A Shack Hartmann (SH) wavefront sensor [1] basically consists on a microlens array and a CCD camera (Fig.1.). The wavefront is sampled by the microlens array in such a way that each sampled light pencil is brought to a particular location in the focal plane of the array. If the wavefront is aberrated, these spots shift from the regularly spaced ideal spot pattern produced by a plane wavefront. These displacements provide information on the local slope of the wavefront over each microlens, and then the WA can be reconstructed using suitable software. Fig. 1 shows a simple scheme of the SH wavefront sensor basic working principle.

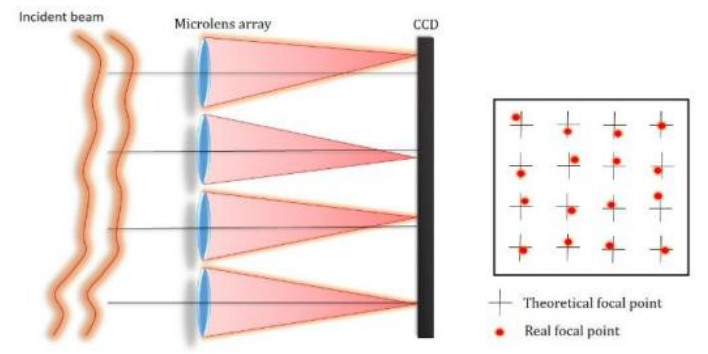

Fig.1. Schematic of a Shack-Hartmann wavefront sensor when an aberrated wavefront reaches the microlens array.

When SH is associated to adaptive optics all this information of the WA is used to manipulate the wavefront with adaptive optical elements like a deformable mirror which provides precise control of the shape of the wavefront. This is why adaptive optics is employed in a broad range of imaging and non-imaging applications to reduce aberrations, improve image quality or shape laser beams, and its intensive use in areas of astronomy and opftalmology.

As is broadly shown in bibliography $[5,6,7]$ the wavefront aberration function $W(\rho, \theta)$ of an optical system can be expressed as a function of a complete set of polynomials. There are many sets of polynomials that fit this properties, but there is one that is very useful and commonly used on the study of optical systems, introduced by Fritz Zernike in 1934. There are more details about in the fifth section.

\subsection{Point Diffraction Interferometer}

The Point Diffraction Interferometer PDI is not an extended and popular tool as the SH, but in the last few years this has been proposed and used in analysis of ocular aberrations. The PDI is a simple common-path interferometer used to directly measure optical path differences, and basically consists on a semitransparent plate with a clear pinhole, as shown in Fig.2.

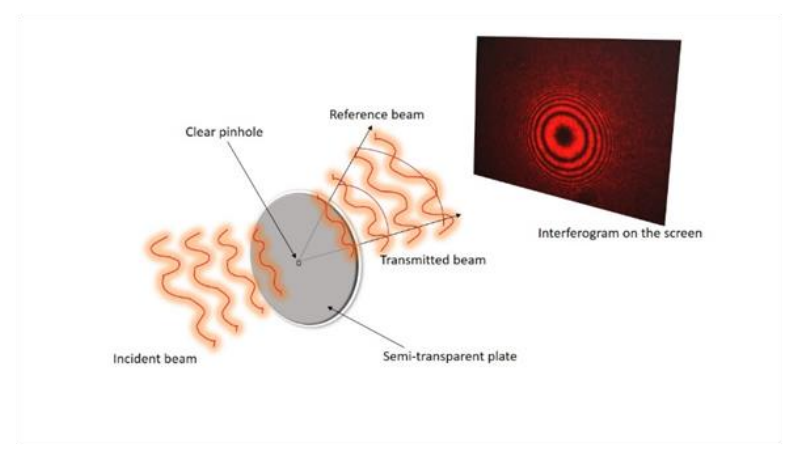

Fig. 2: Basic principle of a PDI.

When a light beam reaches the plate, a spherical reference wave is produced by diffraction at the clear pinhole while the rest of the beam passes through the plate without any change in phase. If the size of the pinhole and the transmittance of the plate are chosen in such a way that both beams have similar amplitude, well contrasted fringes will be observed in any plane placed after the plate $[4,8]$ which can be captured obtaining an interferogram. With a suitable processing of the interferogram it is possible to reconstruct the waveform with all its Zernike coefficients and different features. 


\section{Application of $\mathbf{S H}$ for non contact quality analysis in antique instruments}

The best way to test the performance of an optical instrument is to use it in the conditions and circumstances for which it has been designed. But this situation is not always possible in the case of delicate instruments or old and valuable ones belonging to museums, heritage collections or other special cases.

We have proposed for those cases the use of a Shack-Hartmann wavefront sensor placed at the exit of the instrument in combination with a suitable illumination [9]. So, using the experimental parameters provided by the SH analyzer (Zernike polynomial coefficients) and our own software we are able to calculate the Point-Spread Function (PSF) of the instrument that we are studying, and then calculate the convolution within the PSF and the function that describes a paraxial image. In other words, without removing the instrument from the museum, we are able to see the images as they would have been seen in their time.

Using Fresnel diffraction framework, the complex amplitude of the PSF, $\mathrm{h}(\mathrm{x}, \mathrm{y}, \mathrm{z})$, is defined and calculated as [10]:

$$
h(x, y, z) \propto \frac{1}{\lambda z} \int_{E P} \exp \left[\operatorname{ikW}\left(x_{0}, y_{0}\right)\right] \exp \left[-i k \frac{\Delta z}{2 z^{2}}\left(x_{0}^{2}+y_{0}^{2}\right)\right] \cdot \exp \left[-i \frac{k}{z}\left(x x_{0}+y y_{0}\right)\right] d x_{0} d y_{0}
$$

Where $\mathrm{W}\left(\mathrm{x}_{0}, \mathrm{y}_{0}\right)$ is the WA at the exit pupil EP, which has the coordinates $\left(\mathrm{x}_{0}, \mathrm{y}_{0}\right)$ normalized at its edge, and $\mathrm{k}$ is the wavenumber, defined as: $\mathrm{k}=2 \pi / \lambda$. The second exponential term provides information of defocus $\Delta \mathrm{z}$ in the observation plane, and the last exponential is just the Fourier transform kernel. It is possible to measure the WA in another plane and then use this framework to retro-propagate the PSF through z-axis [11]. Moreover, convolving the PSF with an image it is possible to simulate how a real image could be seen through the optical system under study [9].

The instrument used was an extensible terrestrial telescope manufactured by the British company T.T. \& H. Ltd in 1916, comprising four extension brass tubes. At the larger end there is the objective composed of an achromatic doublet of $50 \mathrm{~mm}$ aperture and approximately $500 \mathrm{~mm}$ of focal length.

Fig. 3 shows the schematic of the experimental setup.

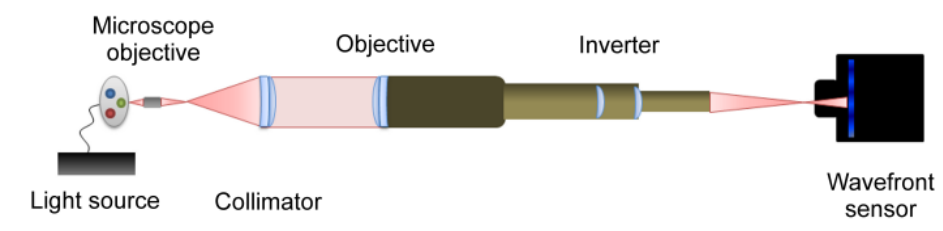

Fig. 3. Schematic of the experimental setup for testing an antique terrestrial telescope.

What it is important in this study is to fit all the exit beam into the SH pupil in order to obtain a good measurement. Thus, the important fact was to capture the whole wavefront in order to calculate and evaluate the evolution of the PSF at the focal region.

This measurement provides a set of Zernike coefficients for each wavelength which were introduced in our own software in order to simulate the evolution of the PSF.

In order to show that the visual appearance of the object through the antique instrument and the simulated image were similar Fig. 4-reproduce the results of the implementation of our method. In the first row we see the capture corresponding to the Gaussian image plane (i.e., the perfect paraxial image); in the second row, the images of the objects seen through the tested instrument at the best visual image plane and two more at planes obtained by moving the last tube of the telescope approximately $0.5 \mathrm{~mm}$ before and after the best visual image plane. Finally, in the third row there are presented the simulations using experimental data provided by the SH measurements and our own software [9]. Real images and the simulated ones agree very well. In the experimental results, the influence of the chromatism was clearly observed by seeing bluish and reddish contours of fine details, which changed by refocusing the images. This effect is also observed in the simulation of the images. 
(a)

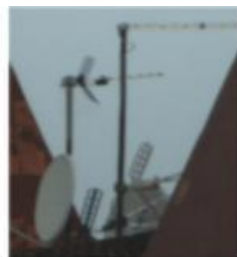

(b)
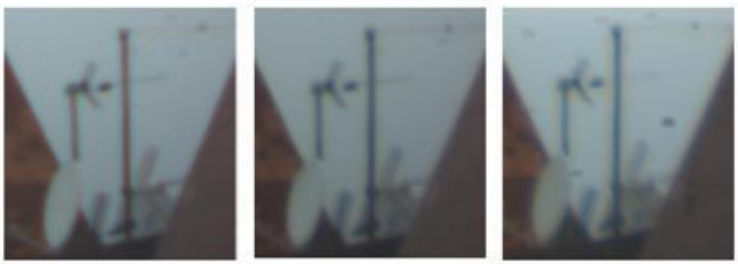

(c)
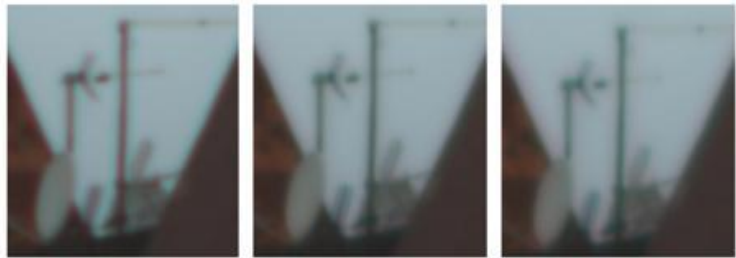

Fig. 4. Results of the application of our method: (a) reference image, (b) real images seen through the antique telescope and (c) simulated images of the reference image.

In a similar way we can simulate the image obtained by a microscope. We are carrying on the study of the image obtained by an objective of an antique microscope made by the French maker Chevalier at the last quarter of the nineteen Century.

\section{Application of PDI for measurement of caustics of crystalline lenses of fishes.}

The study of caustics is important because they contain information about the image formation properties of optical systems $[12,13]$. We use the concept of caustic as a set of focal points, and we have developed a second order approach theory to determine local slopes and curvatures of a wavefront emerging from an optical system. The method of analysis of the wavefront that exits the optical system is based on the use of a Point Diffraction Interferometer [14]. The resulting interference is recorded and the analysis of the interferograms allows us to compute the focal region.

We have applied this interferometric technique for the measurement of local curvatures and caustics of noisy wavefronts such as those produced by biological samples, specifically in crystalline lenses of fish eyes. The study is now focused on the influence of different wavelengths in order to correlate with the spectral sensitivity to different types of cones present in some species of fishes [15].

An eye lens of a scorpion fish (Scorpaena scrofa) was placed in a glass cell filled with supplemented Medium 199 plus solution to prevent fast degeneration. The lens was excised a few hours postmortem. Fig. 5 shows the basic scheme of this setup.

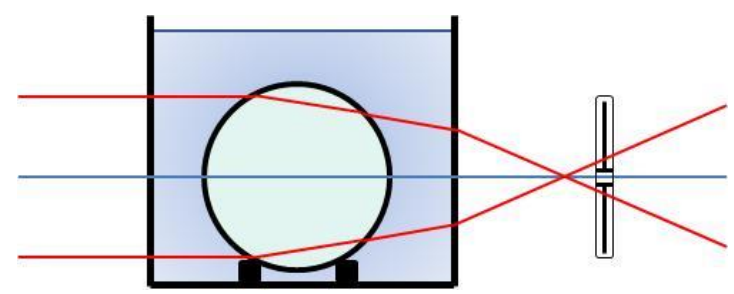

Fig. 5. Detail of the set up for the measurement of the properties of eye lenses. Glass cell with the crystalline lens illuminated by a plane wave and PDI plate in the vicinities of the focal region of the fish eye lens.

Considering Fig. 6, let be $R_{x}$ and $R_{y}$ respectively the distance from a point $\left(x_{0}, y_{0}\right)$ of the WA plane to the sagittal focal line and the tangential focal line along the ray with direction cosine $\alpha_{0}$ passing through $\left(x_{0}, y_{0}\right)$.

In order to obtain what we call the experimental caustic we fit the first dark fringe of the interferograms to an ellipse in order to obtain the values for the major and minor axis. From the height of the pinhole, the center of the ellipses $y_{0}$ and the distance from the pinhole to the observation plane we calculate $\alpha_{0}$ and 
therefore $R_{x}$ and $R_{y}$. Due to the symmetry the scanning is performed for only positive or negative values of the height of the PDI $y_{p}$. Fig. 7 illustrates more clearly the relationship between the pinhole position and the center of the ellipses as explained above.

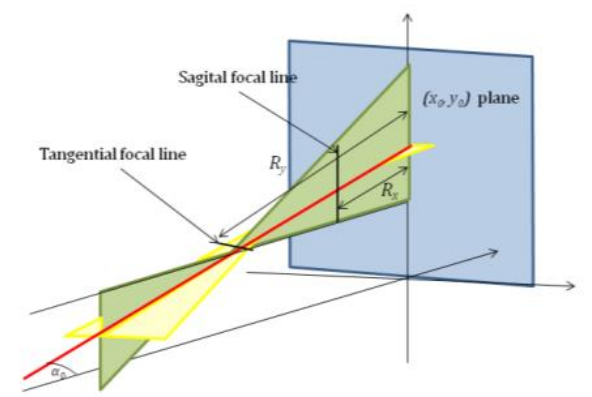

Fig. 6. Ray tracing equivalent.

The PDI plate is displaced vertically to allow scanning of the local curvatures and the slope of the phase across the observation plane. From the size of the axes of the first dark ellipse, the local curvatures (or equivalently $R_{x}$ and $R_{y}$ ) are obtained, and the slope is determined by controlling the position of the pinhole. In this case, in order to check the symmetry of the lens, the scanning is done to obtain both upper and lower branches of the tangential caustic surface. With all these data, the position of the corresponding points at the caustic surfaces is evaluated as explained below. Fig. 8 shows the results for tangential and sagittal caustics.

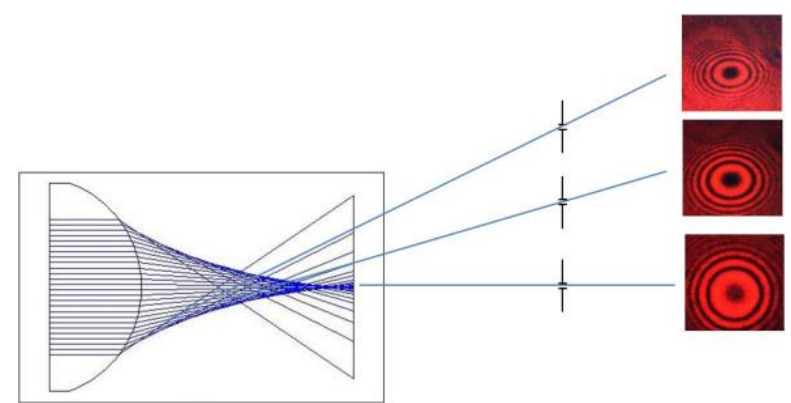

Fig. 7. Scanning of the phase at the observation plane by moving the pinhole transversally.

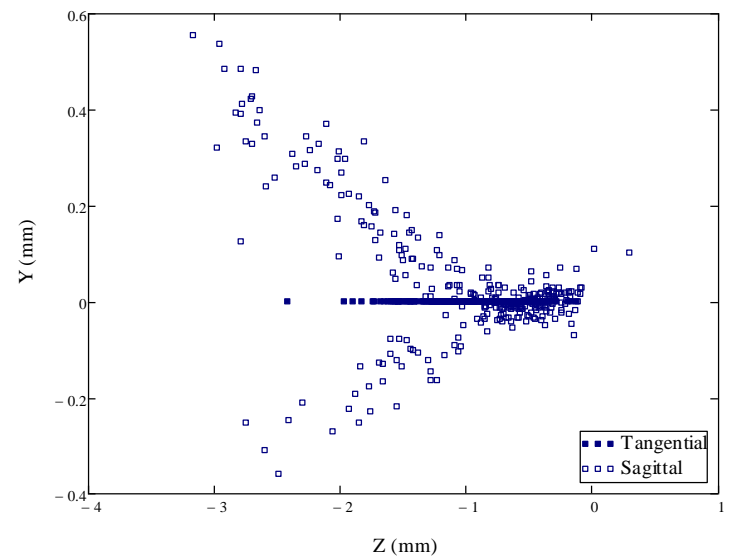

Fig. 8. Tangential and sagittal caustics of fish crystalline lens.

\section{Comparative study of both techniques}

In this section a comparative study between those two techniques on the analysis of a meniscus lens is performed. A similar equivalence has been reported before by Bueno et al. [16] on the study of phase plates but not in the case of real focusing systems. The system under test was a meniscus lens, whose geometrical and optical properties are shown in Table I. A simple scheme of the lens is shown in Fig. 9.

The basic experimental setup consists on an He-Ne laser $(\lambda=633 \mathrm{~nm})$ as a source (S), an achromatic doublet with a focal length of $500 \mathrm{~mm}$ working as a collimator (C) and our meniscus lens (L) in an optical bench. 
TABLE I: Optical and geometrical parameters of the meniscus lens under test: $n$ is the refractive index, $\mathrm{R}_{1}$ and $\mathrm{R}_{2}$ are the curvature radii, and $e$ is the thickness of the lens. The nomenclature is the same presented in Fig. 9.

\begin{tabular}{|l|l|l|l|}
\hline $\boldsymbol{n}$ & $\mathbf{R}_{\mathbf{1}} \mathbf{( m m )}$ & $\mathbf{R}_{\mathbf{2}} \mathbf{( m m )}$ & $\mathbf{e}(\mathbf{m m})$ \\
\hline 1.5151 & 59.0 & 128.9 & 5.4 \\
\hline
\end{tabular}

The semitransparent plate of the PDI that we used in this work was made by coating a glass substrate with Cr. The optical density was 2.5 and the pinhole diameter was $15 \mu \mathrm{m}$.

The SH device used in this work was a HASO32 $2^{\mathrm{TM}}$ from the Imagine Optics Company. The microlenses array consists on $32 \times 32$ microlenses of $6 \mathrm{~mm}$ focal length and a 512x512 pixels CCD sensor.

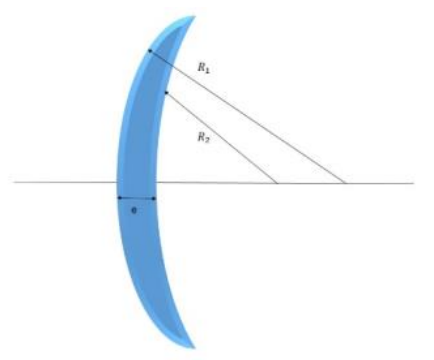

Fig. 9. Scheme of the meniscus lens under test

In order to compare both techniques described before, it was crucial that the observation plane and the pupil size were the same for both measurements. So, obtaining the same experimental conditions for each measurement was the key point of this experiment. This consists basically to make the microlenses array plane of the SH the same of the screen of the PDI. A scheme of the experimental setup using both techniques is shown in Fig.10.

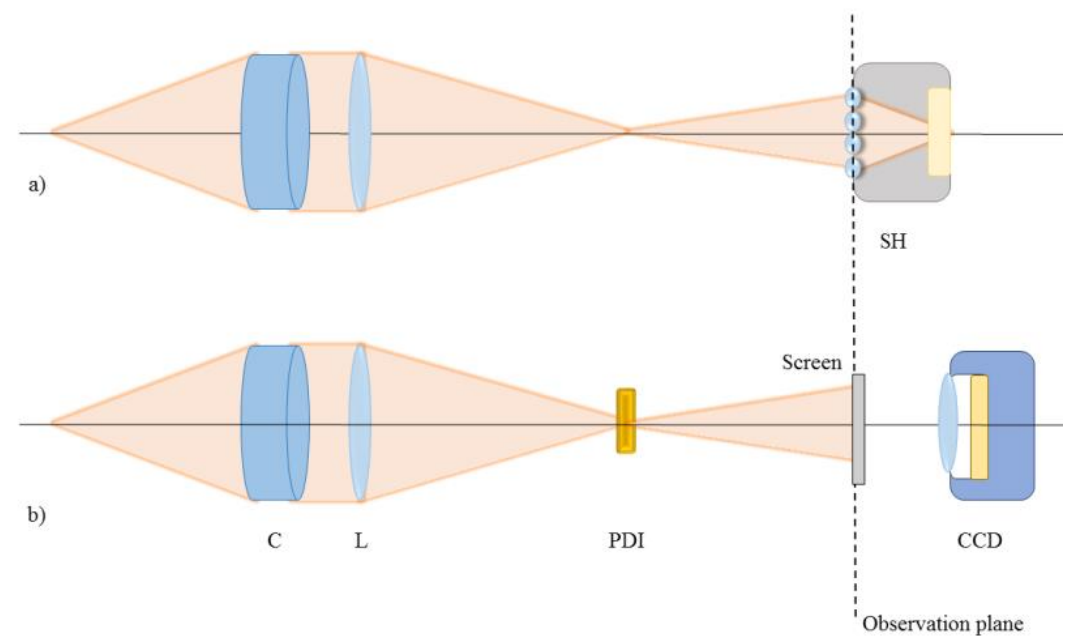

Fig. 10. Dual experimental setup used in this work. a) Schematization of the configuration for the analysis using SH wavefront sensor. From left to right: collimator system (C), lens under test (L) and Shack-Hartmann wavefront sensor (SH). b) Schematization of the setup using the PDI. As it can be seen, for this schematic there are the same components but the SH is replaced in or der to use the PDI, a screen to project the interferogram and a CCD camera to record this interferogram. The observation plane of the screen is the same of the focal plane of SH wavefront sensor. The source used was a He-Ne laser.

So for an observation plane defined by the best operation configuration for the PDI, i.e. the plane that provides the higher number of fringes with best contrast in the interferogram, some captures of the interferograms are taken. For the SH technique, the PDI configuration is replaced by the wavefront sensor and several measurements with this instrument are taken. Since the purpose of the work is to compare the two methods, the measures have been taken in the above-mentioned plane. To measure the lens aberrations, a well corrected optical system must be added to project the lens pupil onto the sensors.

For the image processing of the interferograms the Atmosfringe ${ }^{\circledR}$ software was used [17]. Placing a circumference with the SH pupil measured radius in the interference picture, the software could reconstruct 
the wavefront measured at the observation plane tracing isophase curves. Then, Zernike coefficients are computed and could be compared with the ones provided by the SH wavefront sensor.

In order to verify the simulations of the calculated PSF's, the experimental ones were captured by using a CCD camera.

We have used Zernike polynomials, which expressed in polar coordinates ( $\rho$ and $\theta$ ) are orthogonal in the unit circle. This is a very useful property because it means that excluding any term of the expansion it would not modify the adjustment between the analytical expression of WA and the real aberrated wavefront. So, using the Zernike polynomials the WA, $W(\rho, \theta)$ could be write as [7, 9]:

$$
W(\rho, \theta)=\sum_{\mathrm{n}=0}^{\infty} \sum_{m=0}^{n} C_{n m} Z_{n}^{m}(\rho, \theta)
$$

Where $\mathrm{C}_{\mathrm{nm}}$ corresponds to the Zernike coefficient, or term, and $\mathrm{Z}_{\mathrm{n}}^{\mathrm{m}}(\rho, \theta)$ is the corresponding function of the polynomial expansions.

As was mentioned before, the aim of this study was to compare the PDI and SH wavefront sensor measurements for a meniscus lens. In order to establish a comparison criterion, we have used the rootmean square, RMS, wavefront error of a Zernike expansions $[6,7,16]$ which is defined as follows:

$$
R M S=\left(\sum_{j=4}^{N} C_{j}^{2}\right)^{\frac{1}{2}}
$$

Where $C_{j}$ are the Zernike coefficients, $N$ is the number of the coefficients used in the expansion and $j$ is an index that considers $n$ and $m$. The $j$ index starts on 4 because the first 3 terms $(0,1$ and 2$)$ are the pseudoaberrations of piston and tilts, which have no relevant information about WA [16], and the term $C_{3}$ represents the defocus aberration [16], which was removed in order to enhance the higher order aberrations which were the objective of this work.

It is important to remark that when the PDI method is used, the term of defocus can be evaluated by the distance between the pinhole and the paraxial focus whereas with $\mathrm{SH}$ the term of defocus is directly measured. As we are regarding to the evaluation of the high order aberrations we do not take into account this term for this calculation.

Using this procedure, we reconstructed the aberrated wavefront using both techniques and then, using (1) and (2) we have computed the corresponding PSF and then retro-propagated it. Fig. 11 and Fig. 12 show the results of these calculations, which agree very well with the corresponding experimental PSF at each plane. As it was supposed, the so-called spherical aberration is dominant in the points corresponding to the planes perpendicular to z-axis that were considered in this study.

The comparison shows that both techniques provide quite similar results. In the third row of Fig. 12 a small lack of exposition prevented the view of some rings. Moreover, the important fact is that the evolution of the PSF around the focal region follows a similar behavior. Particularly, the resulting difference RMS between both techniques (from $4^{\text {th }}$ to $8^{\text {th }}$ order in the expansion) was $0.06 \mu \mathrm{m}$.
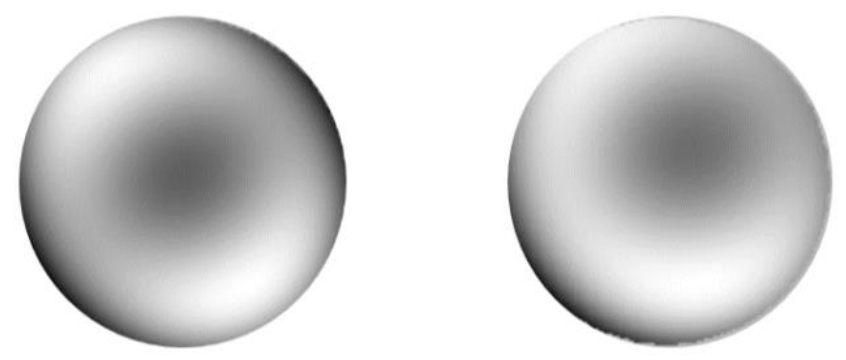

Fig. 11. Resulting WA map for SH (left) and PDI (right) measurements. Tilt and defocus are excluded. 

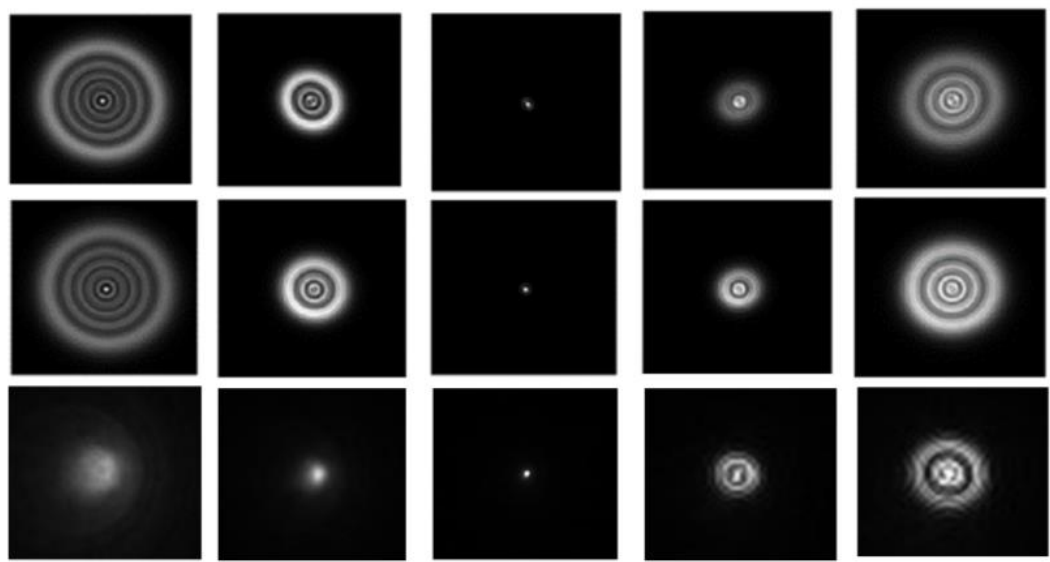

Fig. 12. Retropropagation of WA's PSF in the vicinities of the focal plane for SH (first row) and PDI (second row) measurements. Third row shows the experimental PSF at the same set of planes captured in the laboratory using the CCD camera. A visual inspection shows that both techniques agree: and that the experimental PSF shows a similar trend.

To appreciate the similarity of the reconstructed wavefronts in another way, a computational experiment was though: convolve the calculated PSFs with a perfect image in order to evaluate how the aberrations of the system affects to the image formation.

To do this final test, Lenna's image was used. Fig. 13 shows the convolution of SH and PDI PSF's with Lenna. It is clearly that the resulting image is practically the same in both cases.
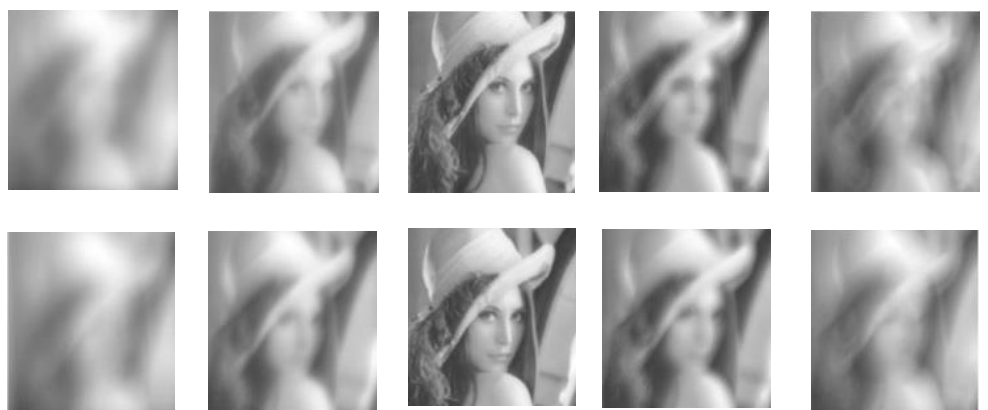

Fig. 13. Results of the convolution between Lenna's image and the PSF's obtained in Fig. 12. First row corresponds to the simulation done with the SH set of PSF's and the second row to the PDI's ones.

\section{Conclusions}

A comparative study between those two different techniques for characterizing wavefronts, on the analysis of a meniscus lens has been carried out. Equivalence between both techniques has been tested and demonstrated, obtaining a difference between the associated RMS corresponding to both techniques of 0.06 $\mu \mathrm{m}$. Two different applications developed by our group with promising results are also described.

On the one hand, the use of PDI is a very simple technique. The principal problem arises in the image processing of the interferogram by the fact that depending on plate location it could introduce noise in the measurement.

On the other hand, the SH is a robust and well-known device used in the study of optical aberrations. It presents an advantage over the PDI as it provides a more accurate reconstruction of the WA, a real time alignment capability and no need to use coherent light. But it presents the drawback that it is not possible to detect variations smaller than the size of the microlenses, and when the relative aperture is large, precision diminishes. Although we only have shown a few applications of these techniques there are a vast range of possibilities.

\section{Acknowledgements}

This work was supported by the "Agencia Estatal de Investigación" (AEI) and the "Fondo Europeo de Desarrollo Regional" (FEDER), under Project FIS2016-77319-C2-2-R of the Spanish "Ministerio de Economía, Industria y Competitividad". 